Journal of Mathematics and Informatics

Vol. 10, 2017, 141-148

ISSN: 2349-0632 (P), 2349-0640 (online)

Published 11 December 2017

www.researchmathsci.org

DOI: http://dx.doi.org/10.22457/jmi.v10a19

Journal of

Mathematics and

Informatics

\title{
Observations on the Homogeneous Ternary Cubic Equation with Four Unknowns $3\left(x^{3}+y^{3}\right)=2 z w^{2}$
}

\author{
J.Kiruthika ${ }^{1}$ and T.R.Usha Rani ${ }^{2}$ \\ ${ }^{1}$ Department of Mathematics, Shrimati Indira Gandhi College \\ Trichy-2, Tamilnadu, India \\ 1e-mail:kiruthi.jj@gmail.com; ${ }^{2}$ e.mail:usharanisigc@gmail.com
}

Received 4 November 2017; accepted 7 December 2017

Abstract. The homogeneous ternary cubic equation given by $3\left(x^{3}+y^{3}\right)=2 z w^{2}$ is analysed for its non-zero distinct integer solutions. A few interesting relations between the solutions and special polygonal and pyramidal numbers are presented.

Keywords: homogeneous cubic, ternary cubic, integer solutions, polygonal numbers, pyramidal numbers.

AMS Mathematics Subject Classification (2010): 11D25

\section{Introduction}

The Diophantine equation offer an unlimited field for research due to their variety [1-3]. In particular, one may refer [4,5] for cubic equations with three unknowns. In [6-8] cubic equations with four unknowns are studied for its non-trivial solutions. This communication concerns with the problem of obtaining non-zero integral solutions of cubic equation with four variables given by $3\left(x^{3}+y^{3}\right)=2 z w^{2}$. A few properties among the solutions and special numbers are presented.

\section{Notations}

$t_{m, n}=n\left[1+\frac{(n-1)(m-2)}{2}\right]$ - Polygonal number of rank $\mathrm{n}$ with sides $\mathrm{m}$

$C t_{m, n}=\frac{m n(n-1)+2}{2}-$ Centered polygonal number of rank $\mathrm{n}$ with sides $\mathrm{m}$

$S_{n}=6 n(n-1)+1-$ Star number of rank $\mathrm{n}$

$P R_{n}=n(n+1)$ - Pronic number of rank $\mathrm{n}$

$G_{n}=2 n-1-$ Gnomonic number of rank $\mathrm{n}$

$j_{n}=2^{n}+(-1)^{n}-$ Jacbosthal-Lucas number of rank $\mathrm{n}$ 
J.Kiruthika and T.R.Usha Rani

\section{Method of analysis}

The cubic Diophantine equation with four unknowns to be solved is given by

$$
3\left(x^{3}+y^{3}\right)=2 z w^{2}
$$

The substitution of the linear transformations

$x=u+v, y=u-v, z=3 u, u \neq v \neq 0$

in (1) leads to

$u^{2}+3 v^{2}=w^{2}$

(3) is solved through different approaches and the different patterns of solutions of (1) obtained are presented below.

\subsection{PATTERN 1}

Assume $w=a^{2}+3 b^{2}$

Write (3) as

$(u+i \sqrt{3} v)(u-i \sqrt{3} v)=[(a+i \sqrt{3} b)(a-i \sqrt{3} b)]^{2}$

Consider the positive factor

$$
u+i \sqrt{3} v=a^{2}+i 2 \sqrt{3} a b-3 b^{2}
$$

Equating real and imaginary parts

$$
\begin{aligned}
& u=a^{2}-3 b^{2} \\
& v=2 a b
\end{aligned}
$$

Substituting u,v in (2), we obtain the non-zero distinct integral solutions of (1) as

$$
\begin{aligned}
& x(a, b)=a^{2}-3 b^{2}+2 a b \\
& y(a, b)=a^{2}-3 b^{2}-2 a b \\
& z(a, b)=3 a^{2}-9 b^{2} \\
& w(a, b)=a^{2}+3 b^{2}
\end{aligned}
$$

\section{PROPERTIES}
1. $z(a, b)+3 w(a, b)-3 t_{6, b} \equiv 0(\bmod 3)$
2. $z(1, n)+18 t_{3, n}-3 \equiv 0(\bmod 9)$
3. $6\left\lfloor y(a, b)+4 t_{4, b}\right\rfloor$ is a nasty number
4. $z\left(2^{n}, 2^{n}\right)+6 j_{2 n}+4=0$
5. $w\left(2^{n}, 2^{n}\right)-4 j_{2 n}+4=0$

\subsection{PATTERN 2}

Assume $w=\left(a^{2}+3 b^{2}\right) * 1$

Write ' 1 ' as

$$
1=\frac{(1+i \sqrt{3})(1-i \sqrt{3})}{4}
$$


Observations on the Homogeneous Ternary Cubic Equation with Four Unknowns $3\left(x^{3}+y^{3}\right)=2 z w^{2}$

Substituting (4) and (5) in (1) and employing the method of factorization, we get $(u+i \sqrt{3} v)(u-i \sqrt{3} v)=\frac{(1+i \sqrt{3})(1-i \sqrt{3})}{4} *[(a+i \sqrt{3} b)(a-i \sqrt{3} b)]^{2}$

Consider,

$$
u+i \sqrt{3} v=\frac{(1+i \sqrt{3})}{2}(a+i \sqrt{3} b)^{2}
$$

Equating real and imaginary parts of the above equation, we get

$$
\begin{aligned}
& u=\frac{a^{2}-3 b^{2}-6 a b}{2} \\
& v=\frac{a^{2}-3 b^{2}+2 a b}{2}
\end{aligned}
$$

Assume $a=2 A, b=2 B$ in the above equations and in view of (2), we obtain the non -zero distinct integral solutions of (1) as

$$
\begin{aligned}
& x(A, B)=4 A^{2}-12 B^{2}-8 A B \\
& y(A, B)=-16 A B \\
& z(A, B)=6 A^{2}-18 B^{2}-36 A B \\
& w(A, B)=4 A^{2}+12 B^{2}
\end{aligned}
$$

\section{PROPERTIES}
1. $2 x(A, B)-y(A, B)-8 t_{4, n}+12 t_{6, B} \equiv 0(\bmod 12)$
2. $z(A,-A)-24 P R_{A}+12 G_{A}+12=0$
3. $w\left(2^{n}, 2^{n}\right)-16 j_{2 n}+16=0$
4. $6[(w(A, A))]$ is a Nasty number
5. $x(A, A)-y(A, A)-32 t_{4, n}=0$

\subsection{PATTERN 3}

Assume $w=\left(a^{2}+3 b^{2}\right) * 1$

' 1 ' can also be written as

$$
1=\frac{(1+i 4 \sqrt{3})(1-i 4 \sqrt{3})}{49}
$$

Substituting (6) and (7) in (1) and employing the method of factorization, we get

$$
(u+i \sqrt{3} v)(u-i \sqrt{3} v)=\frac{(1+i 4 \sqrt{3})(1-i 4 \sqrt{3})}{49} *[(a+i \sqrt{3} b)(a-i \sqrt{3} b)]^{2}
$$

Consider the positive factor

$$
u+i \sqrt{3} v=\frac{1+i 4 \sqrt{3}}{7}(a+i \sqrt{3} b)^{2}
$$

Equating real and imaginary parts on both sides and assume $a=7 A, b=7 B$, we get 
J.Kiruthika and T.R.Usha Rani

$$
\begin{aligned}
& u=7 A^{2}-21 B^{2}-168 A B \\
& v=28 A^{2}-84 B^{2}+14 A B
\end{aligned}
$$

Substituting $\mathrm{u} \& \mathrm{v}$ in (2), we obtain the non-zero distinct integral solutions of (1) as

$$
\begin{aligned}
& x(A, B)=35 A^{2}-105 B^{2}-154 A B \\
& y(A, B)=-21 A^{2}+63 B^{2}-182 A B \\
& z(A, B)=21 A^{2}-63 B^{2}-504 A B \\
& w(A, B)=49 A^{2}+147 B^{2}
\end{aligned}
$$

\section{PROPERTIES}

1. $y(B, B)-x(B, B)-168 t_{3, n} \equiv 0(\bmod 84)$

2. $z(1, n)+63 P R_{n}-21 \equiv 0(\bmod 441)$

3. $y(n, n)+w(n, n)-2 C t_{50, n}-S_{n}+3 \equiv 0(\bmod 56)$

4. $6[w(A, A)]$ is a Nasty number

5. $z\left(2^{n}, 2^{n}\right)+y\left(2^{n}, 2^{n}\right)-322 j_{2 n}+322=0$

\subsection{PATTERN 4}

Consider the linear transformations

$$
\left.\begin{array}{c}
u=\alpha+3 \mathrm{~T} \\
v=\alpha-\mathrm{T}
\end{array}\right\}
$$

Substituting (9) in (3) we get,

$$
\left.\begin{array}{c}
(\alpha+3 T)^{2}+3(\alpha-T)^{2}=w^{2} \\
4 \alpha^{2}+12 \mathrm{~T}^{2}=w^{2}
\end{array}\right\}
$$

Take

$$
w=a^{2}+12 b^{2}
$$

Using (11) in (10), we get

$$
(2 \alpha+i \sqrt{12} T)(2 \alpha-i \sqrt{12} T)=[(a+i \sqrt{12} b)(a-i \sqrt{12} b)]^{2}
$$

Equating the positive factor, we get

$$
(2 \alpha+i \sqrt{12} T)=a^{2}+i \sqrt{12} a b-12 b^{2}
$$

Equating real and imaginary parts

$$
\left.\alpha=\frac{a^{2}-12 b^{2}}{2}\right\}
$$

Substituting (12) in (9), we obtain 
Observations on the Homogeneous Ternary Cubic Equation with Four Unknowns $3\left(x^{3}+y^{3}\right)=2 z w^{2}$

$$
\left.\begin{array}{l}
u=\frac{a^{2}-12 b^{2}+12 a b}{2} \\
v=\frac{a^{2}-12 b^{2}-4 a b}{2}
\end{array}\right\}
$$

To get integer solutions, assume $\mathrm{a}=2 \mathrm{~A}, \mathrm{~b}=\mathrm{B}$ in (13) and hence the non-zero distinct integer solutions of (1) are given by,

$$
\begin{aligned}
& x(A, B)=4 A^{2}-12 B^{2}+8 A B \\
& y(A, B)=16 A B \\
& z(A, B)=6 A^{2}-18 B^{2}+36 A B \\
& w(A, B)=4 A^{2}+12 B^{2}
\end{aligned}
$$

\section{PROPERTIES}
1. $y(A, B)-2 x(A, B)+8 t_{4, A}-12 t_{6, B} \equiv 0(\bmod 12)$
2. $z(A, B)-24 P R_{A}+12 G_{A}+12=0$
3. $y(A, 1)+w(A, B)-9 G_{A}-2 t_{6, A}-12 P R_{B}-9 \equiv 0(\bmod 12)$
4. $x(A, A)+z(A, A)-24 t_{2, A}=0$
5. $6[\mathrm{w}(\mathrm{A}, \mathrm{A})], 6[\mathrm{y}(\mathrm{A}, \mathrm{A})]$ is a Nasty number

\subsection{PATTERN 5}

Introducing the linear transformations

$$
\left.\begin{array}{c}
u=\alpha-3 T \\
v=\alpha+T
\end{array}\right\}
$$

Substituting (14) in (3), we get

Take

$$
\begin{aligned}
(\alpha-3 \mathrm{~T})^{2}+3(\alpha+\mathrm{T}) & =w^{2} \\
4 \alpha^{2}+12 \mathrm{~T}^{2} & =w^{2}
\end{aligned}
$$

Using (16) in (15), we get

$$
(2 \alpha+i \sqrt{12} T)(2 \alpha-i \sqrt{12} T)=[(a=i \sqrt{12} T)(a-i \sqrt{12} T)]^{2}
$$

Equating the positive factor, we get

$$
(2 \alpha+i \sqrt{12} T)=a^{2}-12 b^{2}+i 2 \sqrt{12} a b
$$

Equating real and imaginary parts, we get

$$
\left.\begin{array}{c}
\alpha=\frac{a^{2}-12 b^{2}}{2} \\
T=2 a b
\end{array}\right\}
$$


J.Kiruthika and T.R.Usha Rani

Substituting (17) in (14), we get

$$
\left.\begin{array}{l}
u=\frac{a^{2}-12 b^{2}-12 a b}{2} \\
v=\frac{a^{2}-12 b^{2}+4 a b}{2}
\end{array}\right\}
$$

Assume $a=2 A, b=2 B$ in (18) and in view (2) the non-zero distinct integer solution of (1) are as follows

$$
\begin{aligned}
& x(A, B)=4 A^{2}-48 B^{2}-16 A B \\
& y(A, B)=-32 A B \\
& z(A, B)=6 A^{2}-72 B^{2}-72 A B \\
& w(A, B)=4 A^{2}+48 B^{2}
\end{aligned}
$$

\section{PROPERTIES}

1. Each of the following expressions is a Nasty number

$$
\begin{array}{ll}
\text { i. } & {[z(A,-A)]} \\
\text { ii. } & 6[w(n, n)-x(n, n)-y(n, n)]
\end{array}
$$

2. $x(A, A)+w(A, A)-8 t_{4, n}=0$

3. $x(A, 1)+y(A, 1)+w(A, 1)-8 t_{4, n} \equiv 0(\bmod 48)$

4. $x(A, A)+210 j_{2 n}-210=0$

5. $w\left(2^{n}, n\right)-4 j_{2 n}-44=0$

\subsection{PATTERN 6}

Write (3) as

$$
(w+u)(w-u)=3 v \cdot v
$$

It can be written in the form of ratio as

$$
\frac{v}{w-u}=\frac{w+u}{3 v}=\frac{m}{n}
$$

which is equivalent to the system of double equations

$$
\left.\begin{array}{l}
m u+n v-m w=0 \\
n u-3 m v+n w=0
\end{array}\right\}
$$

Solving (21) by method of cross multiplication, we get

$$
\left.\begin{array}{c}
w=3 m^{2}+n^{2} \\
u=3 m^{2}-n^{2} \\
v=2 m n
\end{array}\right\}
$$

Substituting (22) in (2), the non-zero distinct integer solutions of (1) are given by, 
Observations on the Homogeneous Ternary Cubic Equation with Four Unknowns $3\left(x^{3}+y^{3}\right)=2 z w^{2}$

$$
\begin{aligned}
& x(m, n)=3 m^{2}-n^{2}+2 m n \\
& y(m, n)=3 m^{2}-n^{2}-2 m n \\
& z(m, n)=9 m^{2}-3 n^{2} \\
& w(m, n)=3 m^{2}+n^{2}
\end{aligned}
$$

\section{PROPERTIES}

1. $x(m, n)+y(m, n)+2 t_{4, n}-6 t_{4, m}=0$

2. $z(1, n)+6 t_{3, m}-9 \equiv 0(\bmod 3)$

3. $\left(x\left(\frac{n(n+1)}{2}, n\right)-y\left(\frac{n(n+1)}{2}, n\right)\right)-4 P_{n}^{5}=0$

4. $6[x(m, n)+y(m, n)-2 w(m, n)]$ is a Nasty number

5. $z(m, n)+y(m, n)-12 t_{4, n}=0$

\subsection{PATTERN 7}

Equation (20) can be written as

$$
\frac{3 v}{w-u}=\frac{w+u}{v}=\frac{m}{n}
$$

This is equivalent to the system of double equations

$$
\left.\begin{array}{c}
m u+3 n v-m w=0 \\
n u-m v+n w=0
\end{array}\right\}
$$

Solving (24) by method of cross multiplication, we get

$$
\begin{aligned}
& w=-m^{2}-3 n^{2} \\
& u=3 n^{2}-m^{2} \\
& v=-2 m n
\end{aligned}
$$

Substituting (25) in (2), the non-zero distinct integer solutions of (1) are given by,

$$
\begin{aligned}
& x(m, n)=3 n^{2}-m^{2}-2 m n \\
& y(m, n)=3 n^{2}-m^{2}+2 m n \\
& z(m, n)=9 n^{2}-3 m^{2} \\
& w(m, n)=-m^{2}-3 n^{2}
\end{aligned}
$$

\section{PROPERTIES :}

1. $z(m, n)+3 w(m, n)+12 t_{4, m}+6 \equiv 0(\bmod 12)$

2. $y(m, n)-x(m, n)-4 P R_{m}+2 G_{m}+2=0$

3. $z\left(2^{n}, 1\right)-9 j_{2 n}+12=0$ 
J.Kiruthika and T.R.Usha Rani

4. $x(n, n)-y(n, n)+4 t_{4, n}=0$

5. $[z(m, m)]$ is a Nasty number

\section{Conclusion}

In this paper, an attempt has been made to obtain all possible integer solutions to the homogeneous ternary cubic equation with four unknowns. $3\left(x^{3}+y^{3}\right)=2 z w^{2}$. One may search for other choices of solutions and their corresponding properties.

\section{REFERENCES}

1. L.E.Dickson, History of Theory of Numbers, Vol 2, Chelsea publishing company, New York, (1952).

2. L.J.Mordell, Diophantine Equations, Academic press, London, (1969).

3. R.D.Carmichael, The theory of numbers and Diophantine analysis, New York, Dover, (1959).

4. M.A.Gopalan and S.Premalatha, Integral solutions of $(x+y)\left(x y+w^{2}\right)=2\left(k^{2}+1\right) z^{3}$. Bulletin of Pure and Applied Sciences, 28E (2) (2009) 197-202.

5. M.A.Gopalan and V.Pandichelvi, Remarkable solutions on the cubic equation with four unknowns $x^{3}+y^{3}+z^{3}=28(x+y+z) w^{2} \quad$ Antarctica J. of Maths., 4(4) (2010) 393-401.

6. M.A.Gopalan and B.Sivagami, Integral solutions of homogeneous cubic equation with four unknowns $x^{3}+y^{3}+z^{3}=3 x y z+2(x+y) w^{3}$, Impact. J. Sci. Tec, 4(3) (2010) 53-60.

7. M.A.Gopalan and S.Premalatha, On the cubic Diophantic equations with four unknowns $(x-y)\left(x y-w^{2}\right)=2\left(n^{2}+2 n\right) z^{3}$, International Journal of Mathematical Sciences, 9(1-2) ( (2010) 171-175.

8. M.A.Gopalan and J.Kaliga Rani, Integral solutions of $x^{3}+y^{3}+(x+y) x y=z^{3}+w^{3}+(z+w) z w$, Bulletin of Pure and Applied Sciences, 29E (1) (2010) 169-173. 